\title{
Home sweet home: how do virus specific T cells navigate to the skin?
}

\author{
Anthony L. Cunningham \\ Centre for Virus Research, Westmead Millennium Institute, Westmead, New South Wales, Australia \\ J. Clin. Invest. 110:441-442 (2002). doi:10.1172/JCI200216516.
}

In mucosal immunology, the importance of homing of specific $\mathrm{T}$ and $\mathrm{B}$ lymphocyte memory cells back to their antigen's tissue of origin has long been established (1). Elucidation of the mechanisms of such homing and their role in pathogenesis and immunodeficiency may provide targets for immunotherapy or vaccines (2). Recently, there has been intense interest in lymphocyte homing during the pathogenesis of inflammatory skin diseases, particularly the role of memory $T$ cells carrying the cutaneous lymphocyte-associated antigen (CLA) marker.

\section{Lymphocyte homing to herpetic lesions}

Antigens that precipitate inflammatory skin diseases are transported by dendritic cells to draining lymph nodes, where they encounter the full repertoire of naive $T$ cells entering the lymph node. These $\mathrm{T}$ cells then proliferate and express activation molecules, including CLA, allowing them to circulate to the cutaneous site of antigen origin. In the post capillary venule, adjacent to cutaneous inflammation, $\mathrm{CLA}^{+}$ cells encounter E-selectin on the

\footnotetext{
Address correspondence to: Anthony L. Cunningham, Centre for Virus Research, Westmead Millennium Institute, Darcy Road, PO Box 412, Westmead, New South Wales 2145, Australia. Phone: (61-2) 9845-9005; Fax: (61-2) 9845-9100; E-mail: tony_cunningham@wmi.usyd.edu.au. Conflict of interest: The author received hononaria and expenses while part of the Vaccine Advisory Board for GlaxoSmithKline 1992-2002.

Nonstandard abbreviations used: cutaneous lymphocyte-associated antigen (CLA); herpes simplex virus (HSV); Epstein Barr virus (EBV); cytomegalovirus (CMV).
}

endothelial cell surface and become intermittently tethered. Then, through the upregulation of integrins such as LFA1 or VLA4 and through the interaction of these receptors with endothelial ICAM 1 and VCAM 1, respectively, the lymphocytes undergo firm adhesion and extravasate along a chemokinedirected pathway to the exact site of inflammation (3). These processes have been well described in autoimmune or inflammatory diseases such as psoriasis and atopic dermatitis, in graft-versus-host disease and in cutaneous $\mathrm{T}$ cell lymphomas. It is ironic that these cutaneous homing responses, which occur in an inappropriate manner in those diseases, have not been previously investigated in the setting of local cutaneous infections, which they are well designed to control.

The results presented by Koelle et al. (4) in this issue of the JCI represent a step forward in understanding the pathogenesis of recurrent herpes simplex in particular, and of chronic or latent/reactivating cutaneous viral infections in general. After its reactivation in the neurons of the dorsal root ganglion, herpes simplex virus (HSV) is transported anterogradely to the axon terminus and is then transmitted from the axon terminus to the epidermal keratinocytes. As described below, a distinct sequence of viral and immunologic events then ensues, leading to the recurrence of a herpetic lesion. These same processes probably occur, not just in the formation of active lesions, but even in the foci of asymptomatic shedding, as axon-tokeratinocyte HSV transmission is probably essential for shedding of infectious virus.

\section{Cytokines in the herpetic lesion}

The immunoreactive cells that control the transmitted HSV are the normal constituents of squamous epidermis, keratinocytes and Langerhan cells. They also include infiltrating cells, first predominantly monocyte/macrophages and CD4 lymphocytes, and later predominantly CD8 lymphocytes $(5,6)$. Infection of keratinocytes induces a sequence of chemokine and cytokine secretion similar to that in the whole lesion in vivo: IFN- $\alpha$ and IFN- $\beta$ accumulate first, followed by the $\beta$ chemokines, IL-12, and finally IL-1 and IL-6.

These various factors appear to play distinct roles in controlling viral spread. The $\beta$ chemokines may assist in chemotaxis into lesions of monocytes, as well as CD4 and CD8 lymphocytes. IL-12 may entrain Th1 patterns of cytokine response from HSV antigen-stimulated CD4 (and CD8) lymphocytes and may be especially important in the induction of IFN- $\gamma$ (7). IFN- $\alpha$ and IFN- $\gamma$ synergize to inhibit infection of keratinocytes after transmission from axon termini (8). Interestingly, HSV-1 and - 2 can downregulate $\mathrm{MHC}$ class I expression by epidermal keratinocytes, but this response is reversed by exposure to IFN- $\gamma$, which is produced primarily by infiltrating CD4 lymphocytes (9). CD8 lymphocytes may not fully recognize the infected keratinocytes until MHCI is restored on their surface by the action of IFN- $\gamma$.

HSV probably infects Langerhan cells, as the closely related monocytederived dendritic cells are susceptible to infection in vitro (10). In murine models, inflammatory signals within the lesion induce emigration of infected or antigen-carrying dendritic cells 
through the lymphatics to local lymph nodes and the subsequent priming of CD4 and CD8 lymphocytes (11).

\section{$\mathrm{CLA}^{+}$cells in the control of herpesvirus infections}

As now shown by Koelle et al., the priming of naive CD8 cells in this setting probably also results in the expression of the homing receptor CLA, which interacts with upregulated E-selectin in venular endothelial cells in the lesion. These authors found that $-50 \%$ of HSV-specific CD8 cells in peripheral blood are $\mathrm{CLA}^{+}$, as opposed to $100 \%$ in lesions, suggesting either selective concentration of these cells or upregulation of the marker within the lesions. IL-12 and TGF- $\beta$, which are secreted by keratinocytes, Langerhan cells or infiltrating macrophages, may contribute to CLA upregulation. Likewise, IL1- $\beta$ from keratinocytes and IFN- $\gamma$ from prior infiltrating CD4 lymphocytes probably upregulate E- selectin in the lesions (6).

Other receptors, which may be necessary for the subsequent migration of CD8 lymphocytes into the inflamed lesion, remain to be discovered. Similarly, it is possible that other subsets of CD8 cells in peripheral blood possess homing receptors distinct from CLA that allow them to target HSV-infected neurons in the dorsal root ganglion rather than skin, but such receptors have not yet been described. Future studies will also be required to address whether the proportion of $\mathrm{CLA}^{+}$ $\mathrm{HSV}$-specific CD8 cells increases in the circulation during the development or reactivation of herpetic lesions or during periods of asymptomatic shedding. The homing receptors for HSV-specific CD4 lymphocytes also need to be defined, as does the mechanism by which the successive infiltration of CD4 and CD8 lymphocytes is controlled.

A final question concerns the role of $\mathrm{CLA}^{+} \mathrm{CD} 8$ cells in controlling infections by other herpesviruses, in particular the Epstein Barr virus (EBV) and cytomegalovirus (CMV), which were used as controls by Koelle et al. (4). HSV is shed from squamous epithelium in the whole female genital tract. There is a marked gradation in the nature of this epithelium, from the moist noncornified vaginal mucosa, which is bathed in antibodies and immune cells, to the dry, fully cornified vulval epithelium. Do the homing receptors on CD8 cells differ for these two sites, as has been suggested by studies with attenuated Simian immunodeficiency virus vaccine candidates (12)? It may be significant in this regard that CLA expression differs among CD8 lymphocytes specific for EBV, CMV, and HSV, viruses that are shed from adjacent sites in the orolabial and cervico-vaginal epithelia. Are such expression patterns relevant to the recently reported sex-specific differences in HSV glycoprotein D vaccine efficacy (13)? More importantly can T lymphocyte homing be exploited to enhance the efficacy of such a vaccine?
1. Butcher, E.C., et al. 1996. Lymphocyte homing and homeostasis. Science. 272:60-66.

2. Czerkinsky, C., et al. 1999. Mucosal immunity and tolerance: relevance to vaccine development. Immunol. Rev. 170:197-222.

3. Robert, C., et al. 1999. Inflammatory skin diseases, T-cells and immune surveillance. N. Engl.J. Med. 341:1817-1828.

4. Koelle, D.M., et al. 2002. Expression of cutaneous lymphocyte-associated antigen by $\mathrm{CD}^{+} \mathrm{T}$ cells specific for a skin-tropic virus. J. Clin. Invest. 110:537-548. doi:10.1172/JCI200215537.

5. Cunningham, A.L., et al. 1985. Evolution of recurrent herpes simplex virus lesions. J. Clin. Invest. 75:226-233.

6. Koelle, D.M., et al. 1998. Clearance of HSV-2 from recurrent genital lesions correlates with infiltration of HSV-specific cytotoxic T lymphocytes. J. Clin. Invest. 101:1500-1508.

7. Mikloska, Z., et al. 1998. In vivo production of cytokines and beta (C-C) chemokines in human recurrent herpes simplex virus lesions - do herpes simplex virus-infected keratinocytes contribute to their production? J. Infect. Dis 177:827-838.

8. Mikloska, Z., et al. 2001. Alpha and gamma interferons inhibit herpes simplex virus type 1 infection and spread in epidermal cells after axonal transmission. J. Virol. 75:11821-11826.

9. Hill, A., et al. 1995. Herpes simplex virus turns off the TAP to evade host immunity. Nature. 375:411-415.

10. Mikloska, Z., et al. 2001. Immature monocytederived dendritic cells are productively infected with herpes simplex virus type $1 . J$. Virol. 75:5958-5964.

11. Mueller, S.N., et al. 2002. Rapid cytotoxic T lymphocyte activation occurs in the draining lymph nodes after cutaneous herpes simplex virus infection as a result of early antigen presentation and not the presence of virus. J. Exp. Med 195:651-656.

12. Cromwell, M.A., et al. 2000. Induction of mucosal homing virus-specific CD8(+) T lymphocytes by attenuated simian immunodeficiency virus. $J$. Virol. 74:8762-8768.

13. Spruance, S.L., et al. 2000. Gender-specific efficacy of a prophylactic SBAS4-adjuvant gD, subunit vaccine against genital herpes disease (GHD): results of two clinical efficacy trials. $40^{\text {th }}$ Interscience Conference on Antimicrobial Agents and Chemotherapy. Toronto, Ontario, Canada. L-6. (Abstr.) 